\title{
Prevalência de sobrepeso e obesidade em pré-escolares e escolares de escolas particulares
}

\author{
Overweight and obesity prevalence in children from private schools
}

\author{
Jéssica Raguza Pazin ${ }^{1}$, Vanessa Saito Donadone ${ }^{1}$, Edeli Simioni de Abreu ${ }^{2}$ \\ Rosana Farah Simony ${ }^{3}$
}

\begin{abstract}
RESUMO
Objetivo: Avaliar o estado nutricional de pré-escolares e escolares de escolas particulares atendidas por uma empresa especializada em alimentação infantil.

Materiais e Métodos: Estudo transversal, com crianças de 2 a 8 anos de idade, matriculadas em três escolas particulares, atendidas por uma empresa especializada em alimentação infantil. Foram aferidos peso e altura e a partir disso, foi calculado o Índice de Massa Corporal (IMC). Os dados foram analisados por meio das Curvas de Crescimento da Organização Mundial da Saúde (2007), utilizando os indicadores Peso/Estatura (P/E), Altura/ldade (A/I), Peso/ldade (P/I) e IMC/Idade (IMC/I).

Resultados: Foram avaliadas 571 crianças de ambos os sexos, sendo que a faixa etária entre 2 a 4 anos foi composta por 396 crianças, com média de idade de 4,1 anos $( \pm 1,35)$. Verificou-se que apesar da maioria $(99,3 \%)$ ter apresentado altura adequada para a idade, os índices P/E e IMC/I mostraram que 32,1 e 29,4\%, respectivamente, das crianças apresentaram risco de sobrepeso.

Conclusão: Os resultados obtidos registram a ausência de deficiências nutricionais importantes na amostra estudada, porém pode-se observar que o excesso de peso se faz presente. Sendo assim, é de suma importância a implantação de ações de educação nutricional tanto na escola como nas famílias, a fim de evitar problemas de saúde na vida adulta.
\end{abstract}

Palavras-chave: antropometria; pré-escolares; criança; transição nutricional.

\begin{abstract}
Objective: To evaluate the nutritional status of preschool and school children from private schools served by a specialized infant feeding company.

Materials and Methods: Cross-sectional study with children aged 2 to 8 years, which attended 3 different private schools. All schools have a specialized company that offers food for the students. Weight and height were measured and the Body Mass Index was calculated. Data were analyzed by the WHO's Child Growth Standards (2007), using the indices weight/height (WHZ), height/age (HAZ), weight/age (WAZ) and BMI/age (BAZ).

Results: 571 children were evaluated, from both genders, in which 396 children were aged between 2 and 4 years, with an average age of 4.1 years $( \pm 1.35)$. We have demonstrated that, although most $(99.3 \%)$ have presented appropriate height for age, the $W H Z$ and $B A Z$ ratios showed 32.1 and $29.4 \%$, respectively, of children with an overweight risk.

Conclusion: The results show the absence of nutritional deficiencies in the sample studied, but it's possible to note that the overweight is present. So, it's very important to implement actions of nutritional education in both home and school, aiming to prevent health problems in adulthood.
\end{abstract}

Keywords: anthropometry; preschool; child; nutritional transition.

\footnotetext{
${ }^{1}$ Nutricionista. Formada pela Universidade Presbiteriana Mackenzie, São Paulo.

${ }^{2}$ Nutricionista. Doutora pela Faculdade de Saúde Pública da USP. Professora do Curso de Nutrição da Universidade Presbiteriana Mackenzie, São Paulo.

${ }^{3}$ Nutricionista. Doutora pela Escola Paulista de Medicina da UNIFESP. Professora do Curso de Nutrição da Universidade Presbiteriana Mackenzie, São Paulo.
} 


\section{INTRODUÇÃO}

O estado nutricional de uma criança possui papel fundamental para que seu crescimento seja progressivo e para que ela desenvolva suas aptidões psicomotoras e sociais. Alterações de déficit ou excesso expõem tais crianças a riscos potenciais de agravos à saúde, e problemas futuros de relações interpessoais $e$ funcionais dentro da comunidade ${ }^{1}$.

A antropometria é amplamente utilizada para avaliação nutricional de indivíduos e de grupos populacionais ${ }^{2}$. O peso e a estatura são as medidas mais utilizadas para avaliação e monitoramento ponderal e estatural durante a infância ${ }^{3}$. Para a classificação do estado nutricional, são necessários padrões de referência e pontos de corte, compondo uma linha divisória, distinguindo os que necessitam e os que não necessitam de intervenção, permitindo determinar o déficit e o excesso de peso ${ }^{4}$.

Em crianças, os índices antropométricos mais frequentemente utilizados são 0 peso/idade, a altura/idade e o peso/altura. Esses índices são obtidos comparando-se as informações de peso, altura, idade e sexo com curvas de referência ${ }^{5}$. Os resultados assim obtidos são expressos como escores Z, percentis ou percentuais da mediana. Para a avaliação do estado nutricional em nível individual, recomenda-se usualmente que os três índices sejam calculados, uma vez que refletem processos diferentes ${ }^{6}$.

Nas últimas décadas, houve um declínio da prevalência de desnutrição em crianças menores de cinco anos de idade ${ }^{7-9}$, porém esta tendência encontrase em um contexto epidemiológico chamado de transição nutricional, no qual há a substituição dos déficits pelos excessos nutricionais ${ }^{10,11}$.

Os distúrbios do estado nutricional e de saúde durante os primeiros anos de vida frequentemente são determinados por diversos fatores. No entanto, nos países em desenvolvimento tais fatores são dependentes das condições gerais de vida e do nível de atendimento das necessidades básicas da população, como renda, escolaridade materna, acesso a programas de assistência à saúde e à creche com o estado nutricional infantil ${ }^{12}$.

No meio urbano, a grande dificuldade das mães em conciliar o emprego com o cuidado infantil impulsionou a criação de creches, as quais constituem um importante fator no perfil de transição nutricional, já que as crianças permanecem na creche oito a dez horas por dia e, durante este tempo, recebem dois terços de suas necessidades nutricionais ${ }^{13}$.

Frente a esta nova realidade associada à vulnerabilidade deste grupo populacional, o objetivo deste trabalho foi realizar a avaliação antropométrica de pré-escolares e escolares de escolas particulares localizadas na cidade de São Paulo.

\section{MATERIAIS E MÉTODOS}

Trata-se de um estudo transversal, com crianças de 2 a 8 anos de idade, de ambos os gêneros, alunos de escolas particulares localizadas na cidade de São Paulo, atendidas por uma empresa especializada em alimentação infantil.

Foram convidadas a participar todos os alunos matriculados nas escolas atendidas pela empresa de alimentação, e que tiveram autorização dos pais ou responsáveis (Termo de Consentimento Livre e Esclarecido), assim sendo não foi necessário realizar o cálculo amostral. Antes da coleta de dados, foi enviada uma carta aos pais pedindo a autorização para aferição dos dados das crianças, aqueles que não responderam à autorização ou negaram a participação não entraram no grupo do estudo.

As medidas comprimento/estatura foram aferidas em duplicata e após treinamento com o nutricionista. A tomada de peso $(\mathrm{kg})$ foi feita com auxilio da balança Filizola $\AA^{\circledR}$, pertencente à empresa em questão, com capacidade para $150 \mathrm{~kg}$ e sensibilidade de $100 \mathrm{~g}$. Os participantes foram pesados com vestimentas leves e sem sapatos. O comprimento/estatura, em centímetros, foi feito utilizando-se um estadiômetro acoplado à mesma balança Filizola $\AA$, pertencente à empresa, com capacidade de $1,98 \mathrm{~m}$ e sensibilidade de $0,5 \mathrm{~cm}$, conforme procedimentos recomendados pela literatura ${ }^{14}$.

As crianças foram avaliadas segundo os índices antropométricos peso/idade $(\mathrm{P} / \mathrm{l})$, estatura (comprimento)/idade (E/I) e peso/estatura (comprimento) $(\mathrm{P} / \mathrm{E})$ e índice de massa corporal/idade 
(comprimento) (P/E) e índice de massa corporal/idade (IMC/I) de acordo com o sexo e a faixa etária, utilizando como referência a distribuição proposta pela Organização Mundial de Saúde em 2006 ${ }^{15}$. Foram classificadas como eutróficas as crianças cujos índices $\mathrm{P} / \mathrm{I}, \mathrm{E} / \mathrm{l}$ e $\mathrm{P} / \mathrm{E}$ foram maiores ou iguais a $-2,0$ escore-z e, em déficit ponderal e/ou estatural, aquelas que apresentaram valores inferiores a $-2,0$ escores-z da mediana da curva de referência. $O$ índice $P / E$ foi utilizado para classificação de sobrepeso, tendo como ponto de corte valores iguais ou superiores a +2 escores-z em relação à estatura ${ }^{2}$. As crianças acima de 5 anos de idade não participam do indicador $P / E$.

Desta forma, foi utilizado para classificação o programa Anthro® $W H O$ para crianças com até 5 anos de idade, e o programa Anthro® WHO Plus para crianças acima de 5 anos de idade, com base na Organização Mundial da Saúde ${ }^{2,15}$. A tabulação dos dados coletados foi organizada pelo programa Excel e contabilizados os indicadores nos programas Anthro $\AA$ WHO e Anthro® WHO Plus, ambos disponíveis no site da Organização Mundial da Saúde. Os resultados foram apresentados em forma de tabelas para melhor visualização. Este trabalho foi aprovado pelo CIEP do Centro de Ciências Biológicas e Saúde da Universidade Presbiteriana Mackenzie (Processo CIEP $\left.\mathrm{n}^{\circ} \mathrm{N} 023 / 12 / 10\right)$.

Para organizar os dados e a tabulação das informações coletadas, foram tabuladas utilizando-se o programa Microsoft Exce® versão 2007. Por se tratar de uma análise exploratória de dados, os mesmos foram abordados por meio da estatística descritiva simples, cujo objetivo básico foi sintetizar uma série de valores de mesma natureza, utilizando-se médias, desvio padrão, frequências e porcentagens. Essas técnicas estatísticas permitiram estabelecer o perfil do grupo quanto à idade, sexo e variáveis antropométricas.

\section{RESULTADOS}

Foram convidadas a participar do estudo 757 crianças com idade de 2 a 8 anos, de ambos os sexos, das escolas atendidas pela empresa de alimentação infantil, sendo que 112 crianças não foram autorizadas pelos pais a participar do estudo. Deste total, 63 crianças
TABELA 1 - Peso para estatura em crianças de 2 a 4 anos de idade, de ambos os sexos, matriculadas em escolas particulares.

\begin{tabular}{|c|c|c|c|}
\hline & Meninos & Meninas & Total \\
\hline Magreza & - & $1(0,5)$ & $1(0,2)$ \\
\hline Eutrofia & $136(68,7)$ & $132(66,7)$ & $268(67,7)$ \\
\hline $\begin{array}{l}\text { Risco de } \\
\text { Sobrepeso }\end{array}$ & $62(31,3)$ & $50(25,2)$ & $127(32,1)$ \\
\hline
\end{tabular}

foram autorizadas a realizar a antropometria, porém devido às faltas e recusas no dia da coleta (10), a amostra final foi de 571 crianças com média de idade de 4,1 anos $( \pm 1,35)$.

A faixa etária entre 2 a 4 anos, foi composta por 396 crianças, uma vez que o indicador $P / E$, não foi aplicado para as crianças acima de 5 anos de idade, devido aos padrões da Organização Mundial da Saúde.

Utilizando-se 0 indicador $\mathrm{P} / \mathrm{E}$, houve uma porcentagem elevada de risco de sobrepeso, aparentemente maior entre o gênero masculino quando comparado com o feminino, conforme mostra a tabela 1 , porém em relação ao indicador $\mathrm{P} / \mathrm{l}$ porcentagem de peso elevado foi menor $(6,36 \%)$, tanto entre meninas $(8,3 \%)$ quanto entre os meninos $(4,9 \%)$ de peso elevado em relação à idade (tabela 2 ).

Analisando o indicador $A / I$, pode-se observar que, com exceção de apenas $0,7 \%$ das crianças (sendo três meninas e um menino), todos estavam com a altura adequada, conforme dados da tabela 3 .

A relação IMC/I indicou que quase $30 \%$ das crianças estavam com risco de sobrepeso, sendo que essa porcentagem foi aparentemente maior entre os meninos $(31,1 \%)$ do que as meninas $(27,8 \%)$ (tabela 4$)$.

\section{DISCUSSÃO}

No presente estudo, a maior parte $(99,3 \%)$ das

TABELA 2 - Peso para idade em crianças de 2 a 8 anos de idade, de ambos os sexos, matriculadas em escolas particulares.

\begin{tabular}{lccc}
\hline Peso /Idade & Meninos & Meninas & Total \\
\hline Muito baixo/baixo & $1(0,4)-$ & $1(0,3)$ & $2(0,4)$ \\
Peso adequado & $268(94,7)$ & $263(91,4)$ & $531(93)$ \\
Peso elevado & $14(4,9)$ & $24(8,3)$ & $38(6,6)$ \\
\hline
\end{tabular}

Dados apresentados como número absoluto (\%). 
TABELA 3 - Altura para Idade em crianças de 2 a 8 anos de idade, de ambos os sexos, matriculadas em escolas particulares.

\begin{tabular}{lccc}
\hline Altura/ldade & Meninos & Meninas & Total \\
\hline Baixa & $3(1,1)$ & $1(0,3)$ & $4(0,7)$ \\
Adequada & $280(98,9)$ & $287(99,7)$ & $567(99,3)$ \\
\hline
\end{tabular}

Dados apresentados como número absoluto (\%).

crianças avaliadas segundo o índice A/l encontrava-se dentro dos padrões de normalidade. Como este índice é considerado o mais sensível para aferir a qualidade de vida da população infantil, isso vai de encontro a amostra avaliada que pertence a escolas particulares $e$ possivelmente não apresentam problemas socioeconômicos, infecções crônicas e recorrentes e aporte nutricional inadequado. Segundo Soares et al. ${ }^{16}$, a maioria das crianças brasileiras (80\%), apresentam baixa estatura. No entanto, não apresentam história de baixo peso ao nascer, o que sugere que essas crianças eram semelhantes à população de referência e que esse déficit surgiu depois do nascimento e, portanto, por fatores não genéticos.

Verificou-se que quase $30 \%$ da amostra estudada apresentou risco de sobrepeso tanto para em relação ao indicador P/E como para o IMC/I. Comparando-se estes resultados com um estudo realizado com pré-escolares na cidade de Duque de Caxias, no Rio de Janeiro ${ }^{1}$, os valores para risco de sobrepeso encontrado no presente segundo a relação P/E foram superiores (32\% vs. 27\%), assim como quando comparado com o estudo de Sotello et al. ${ }^{17}$, realizado em escolas públicas estaduais da cidade de São Paulo com crianças de 6 e 9 anos de idade onde foi verificado a prevalência de $22,2 \%$ de sobrepeso para este índice. Esta diferença pode ser parcialmente justificada pelo fato do trabalho ter sido desenvolvido em escolas particulares, onde o poder aquisitivo é da população é diferente.

Várias pesquisas ${ }^{18-21}$ enfatizam o aumento de peso de crianças nos últimos anos. Cerca de $7 \%$ da população infantil mundial é obesa, e duas a três vezes mais crianças estão com sobrepeso ${ }^{22}$. As maiores taxas de obesidade infantil têm sido observadas em países desenvolvidos, mas a prevalência está cada vez mais elevada nos países em desenvolvimento. Tais dados
TABELA 4 - IMC para Idade em crianças de 2 a 8 anos de idade, de ambos os sexos, matriculadas em escolas particulares.

\begin{tabular}{lccc}
\hline IMC/Idade & Meninos & Meninas & Total \\
\hline Magreza & $3(1,1)$ & $2(0,7)$ & $5(0,9)$ \\
Eutrofia & $192(67,8)$ & $206(71,5)$ & $398(69,7)$ \\
Risco de Sobrepeso & $88(31,1)$ & $80,0(27,8)$ & $168(29,4)$ \\
\hline \multicolumn{2}{l}{ Dados apresentados como número absoluto (\%). }
\end{tabular}

Dados apresentados como número absoluto (\%)

são alarmantes, pois crianças com excesso de peso tendem a ser adultos obesos ${ }^{23}$.

Em um estudo realizado por Fernandes et al. ${ }^{24}$, aproximadamente $1 / 4$ da amostra total estava acima do peso adequado para a idade, fato que se assemelha ao presente estudo, onde $29,4 \%$ das crianças apresentavam risco de sobrepeso (19,8\%), sobrepeso $(6,6 \%)$ ou obesidade (3\%).

De acordo com Guimarães e Barros ${ }^{25}$, o aumento da prevalência de obesidade tem sido observado no Brasil em diferentes áreas e segmentos sociais. Em seu estudo, os autores encontraram a existência de diferenças de perfis nutricionais em crianças de escolas particulares: a justificativa seria a conduta que os pais teriam em relação ao seu filho com os seus hábitos alimentares em casa.

É preciso destacar que o maior risco, em longo prazo, da obesidade infantil, é sua persistência no adulto, com todas as consequências associadas para a saúde ${ }^{26}$.

Os resultados obtidos mostraram a ausência de deficiências nutricionais importantes na amostra estudada, que pode estar ligada às ações das escolas quanto à alimentação das crianças durante o seu período de aula, uma vez que oferecem refeições adequadas e atividades educativas e de lazer que favorecem o crescimento infantil. Entretanto o risco de sobrepeso encontrado pode ser parcialmente explicado pela alimentação inadequada fora da escola. Assim sendo, a promoção da alimentação adequada em casa dever ser também um dos objetivos da educação nutricional nas escolas visto que os hábitos alimentares das famílias podem comprometer o estado nutricional deste grupo etário.

Frente ao exposto, pode-se verificar que a avaliação e diagnóstico nutricional de uma população é uma 
estratégia fundamental para estudo de suas condições de saúde, especialmente em pré-escolares e escolares, uma vez que estes dados podem apontar algumas condições que podem modificar futuramente o estado de saúde das mesmas. A avaliação antropométrica é uma importante ferramenta de diagnóstico nutricional e fundamental para traçar políticas de saúde para a população, e o uso de diferentes indicadores propicia diagnósticos mais seguros não só em relação ao estado nutricional, mas também ao grau de desenvolvimento dos mesmos.

\section{Agradecimentos}

Agradecemos à Nutrical $\circledast$ por possibilitar a realização deste estudo, além das nutricionistas da empresa, que auxiliaram na aferição dos dados coletados.

\section{REFERÊNCIAS}

1. Santos ALB, Leão LSCS. Perfil antropométrico de préescolares de uma creche em Duque de Caxias, Rio de Janeiro. Rev Paul Pediatr. 2008; 26(3):218-24.

2. World Health Organization. Physical status: the use and interpretation of anthropometry. Geneva: WHO; 1995. (WHO - Technical Report Series, 854).

3. Roberts SB, Dalla GE. The new childhood growth charts. Rev Nutr. 2001; 59:31-6.

4. Soares NT. Um novo referencial antropométrico de crescimento: significados e implicações. Rev Nutr. 2003; 16:93-104

5. National Centers For Health Statistics. Growth curves for children, birth - 18 years. Hyattsville: NCHS; 1978. (DHEW(PHS) 78.1650-Series 11, 165).

6. Onís M, Monteiro C, Akré J, Clugston G. The worldwide magnitude of protein-energy malnutrition: an overview from he WHO Global Database On Child Growth. Bull. World Health Organ. 1993; 71:703-12.

7. Brasil. Ministério do Planejamento, Orçamento e Gestão. Instituto Brasileiro de Geografia e Estatística (IBGE). Estudo nacional de despesa familiar. Brasília(DF): IBGE; 1974.

8. Brasil. Ministério da Saúde. Instituto Nacional de Alimentação e Nutrição (INAN). Pesquisa nacional sobre saúde e nutrição: perfil de crescimento da população brasileira de 0 a 25 anos. Brasília (DF): INAN; 1990.

9. Brasil. Ministério do Planejamento, Orçamento e Gestão. Instituto Brasileiro de Geografia e Estatística (IBGE). Pesquisa de orçamentos familiares 2002-2003: antropometria e análise do estado nutricional de crianças e adolescentes no Brasil. Brasília (DF): IBGE; 2006.

10. Kac $\mathrm{G}$, Velásquez-Meléndez $\mathrm{G}$. A transição nutricional e a epidemiologia da obesidade na América Latina. Cad Saude Publica. 2003; 19:S4-5.
11. Oliveira RC. A transição nutricional no contexto da transição demográfica e epidemiológica. Rev Min Saude Pub. 2004; 5:16-23.

12. Monteiro CA. Saúde e Nutrição das crianças de São Paulo: diagnóstico, contrastes sociais e tendências. São Paulo: HUCITEC; 1988.

13. Biscegli TS, Corrêa CE, Romera J, Hernandez JL. Avaliação do estado nutricional e prevalência de carência de ferro em crianças freqüentadoras de uma creche. Rev Paul Pediatr. 2006; 24:323-9.

14. Lohman TG. Advances in body composition assessment: current issues in exercisesscience. Illinois: Human Kinetic Publisher; 1992.

15. World Health Organization. Child growth standards (2006). [Capturado 2011 ago 10]; Disponível em: http://www.who.int/childgrowth/standards/en/.

16. Soares NT et al. Estado nutricional de lactentes em áreas periféricas de Fortaleza. Rev Nutr. 2000; 13(2):99-106.

17. Sotelo YOM, Colugnati FAB, Taddei JAAC. Prevalência de sobrepeso e obesidade entre escolares da rede pública segundo três critérios de diagnóstico antropométrico. Cad Saúde Púb. 2004; 20(1):233-40.

18. Castro TG, Novaes JF, Silva MR, Costa NMB, Franceschini SCC, Tinôco ALA, et al. Caracterização do consumo alimentar, ambiente socioeconômico e estado nutricional de pré-escolares de creches municipais. Rev Nutr 2005; 18:321-30

19. Tremblay MS, Willms JD. Secular trends in the body mass index of Canadian children. CMAJ. 2000; 163:1429-33.

20. Kain J, Uauy R, Vio F, Albala C. Trends in overweight and obesity prevalence in Chilean children: comparison of three definitions. Eur J Clin Nutr. 2001; 56:200-4.

21. Herpertz-Dahlmann B, Geller F, Böhle C, Khalil C, TrostBrinkhues $G$, Ziegler A, et al. Secular trends in body mass index measurements in preschool children from the City of Aachen, Germany. Eur J Pediatr. 2003; 162:104-9.

22. Speiser PW et al. Childhood obesity. J Clin Endocrinol Metab. 2005; 90:1871-87.

23. Dehghan M, Akhtar-Danesh N, Merchant AT. Childhood obesity, prevalence and prevention. Nutr J. 2005; 4:24.

24. Fernandes IT, Gallo PR, Advíncula AO. Avaliação antropométrica de pré-escolares do município de MogiGuaçú, São Paulo: subsídio para políticas públicas de saúde. Rev Bras Saúde Matern Infant. 2006; 6(2):217-22.

25. Guimarães LV, Barros MBA. As diferenças de estado nutricional em préescolares de rede pública e a transição nutricional. J Pediatr. 2001; 77.

26. Guo SS, Roche AF, Chumlea WC, Gardner JD, Siervoge RM. The predictive value of childhood body mass index values for overweight at age 35 years. Am J Clin Nutr. 1994; 59:810-9.

\section{Endereço para correspondência}

Edeli Simioni de Abreu

Alameda São Caetano 857, apto. 43

São Caetano do Sul/SP - CEP 09560-105

Telefone: +5511984349393

E-mail: edeli@mackenzie.com 